\title{
Daytime naps improve motor imagery learning
}

\author{
Ursula Debarnot • Eleonora Castellani • \\ Gaetano Valenza - Laura Sebastiani • Aymeric Guillot
}

Published online: 13 August 2011

(C) Psychonomic Society, Inc. 2011

\begin{abstract}
Sleep is known to contribute to motor memory consolidation. Recent studies have provided evidence that a night of sleep plays a similar functional role following motor imagery (MI), while the simple passage of time does not result in performance gains. Here, we examined the benefits of a daytime nap on motor memory consolidation after MI practice. Participants were trained by MI on an explicitly known sequence of finger movements at 11:00. Half of the participants were then subjected (at 14:00) to either a short nap (10 min of stage 2 sleep) or a long nap (60-90 min, including slow wave sleep and rapid eye movement sleep). We also collected data from both quiet and active rest control groups. All participants remained in the lab until being retested at 16:00. The data revealed that a daytime nap after imagery practice improved motor performance and, therefore, facilitated motor memory consolidation, as compared with spending a similar time
\end{abstract}

U. Debarnot $(\bowtie) \cdot$ L. Sebastiani

Department of Physiological Sciences "G. Moruzzi”,

University of Pisa,

Via San Zeno 31,

56127 Pisa, Italy

e-mail: Ursula.debarnot@gmail.com

U. Debarnot $\cdot$ A. Guillot

Centre de Recherche et d'Innovation sur le sport,

Université Claude Bernard Lyon I,

Université de Lyon, France

E. Castellani

Department of Physiology, University of Siena,

Siena, Italy

\section{G. Valenza}

Interdepartmental Research Centre E. Piaggio,

School of Engineering, University of Pisa,

Pisa, Italy interval in the wake state. Interestingly, the results revealed that both short and long naps resulted in similar delayed performance gains. The data might also suggest that the presence of slow wave and rapid eye movement sleep does not provide additional benefits for the sleep-dependent motor skill consolidation following MI practice.

Keywords Motor imagery - Sleep · Memory consolidation . Daytime nap $\cdot$ Motor sequence learning

\section{Introduction}

A wide range of experimental studies have provided strong evidence that daytime naps contribute to motor memory consolidation in the same manner that a night of sleep (for overviews, see Dhand \& Sohal, 2006; Diekelmann, Wilhelm, \& Born, 2009). Interestingly, nap-related improvements were found to depend on the nature of the task, as well as the stages and duration of sleep (Ficca, Axelsson, Mollicone, Muto, \& Vitiello, 2010). For example, Mednick, Nakayama, and Stickgold (2003) reported that visual discrimination learning improved after a long nap of 60-90 min including both slow wave sleep (SWS) and rapid eye movement (REM) sleep stages. As well, Nishida and Walker (2007) looked for the effect of a similar long nap on a finger-tapping sequence task performance and showed that the amount of delayed gains was correlated with that of sleep stage 2 . In line with these findings, Korman et al. (2007) postulated that 90 min of daytime nap would facilitate memory stability and the expression of delayed gains following finger-tapping tasks.

Another countermeasure that has received growing attention in more recent years is brief napping. Although brief daytime naps rarely contain SWS, since they are 
composed only of sleep stages 1 and 2, naps lasting no more than $10 \mathrm{~min}$ were found to improve alertness and cognitive performance (Takahashi \& Arito, 2000; Tietzel \& Lack, 2001, 2002). Interestingly, Tietzel and Lack (2002) even reported that $10 \mathrm{~min}$ of nap resulted in significantly higher improvement in alertness and cognitive performance, as compared with no nap and ultrabrief naps (30 and $90 \mathrm{~s}$ ). The latter finding might suggest that the mechanisms underlying the benefits of brief naps are not related to the onset of sleep stage 1 but might be elicited by that of stage 2. In the same vein, other studies have confirmed that during a short nap, stage 2 can be required for the enhancement of cognitive performance (Brooks \& Lack, 2006; Hayashi, Watanabe, \& Hori, 1999). So far, it is still unknown whether short naps might contribute to motor memory consolidation as well and whether their benefits would be related to the presence of sleep stage 2 .

Motor imagery (MI) is a dynamic state during which a movement is internally reactivated within working memory and without any motor output (Decety, 1996; Jeannerod, 1994; Malouin, Belleville, Richards, Desrosiers, \& Doyon, 2004). There is now ample evidence that MI contributes to the enhancement of both cognitive and motor performances (for reviews, see Feltz \& Landers, 1983; Guillot \& Collet, 2008). Accordingly, it has been shown that the execution of a movement and MI share several parallel characteristics. There is a certain degree of overlap between the neuronal processes mediating actual and imagined motor performance, and the time course of mentally simulated actions is highly correlated with the time taken to execute the same movement (for reviews, see Guillot \& Collet, 2008; Holmes \& Collins, 2001; Munzert, Lorey, \& Zentgraf, 2009). To date, very few studies based on sleep data and motor skill learning have demonstrated that motor consolidation processes remain effective following MI, hence supporting that a night of sleep after MI practice results in similar memory consolidation than following of the same task (Debarnot et al., 2009; Debarnot, Creveaux, Collet, \& Guillot, 2009; Debarnot, Maley, De Rossi, \& Guillot, 2010). However, these studies did not look at the specific contribution of each sleep stage, since MI consolidation occurring overnight was not controlled by polysomnographic recordings.

On the basis of the findings mentioned above, the present study had two main objectives: (1) to identify whether a nap following MI practice might contribute to improve the motor consolidation process and (2) to determinate which sleep stage might primarily mediate this consolidation process. The experimental protocol included a daytime nap design isolating either the effects of sleep stage 2 (i.e., a short nap including sleep stage 1 and $10 \mathrm{~min}$ in stage 2) or sleep stage 2 along with both SWS and REM (i.e., a long nap lasting $90 \mathrm{~min}$ ). Practically, we used a version of the sequential finger-tapping task developed by Karni et al. (1995) and evaluated motor performance before MI training, as well as before and after a short or long daytime nap. Two other groups in which participants were trained and retested the same day after MI practice (without any intervening nap) were also included to determine the role of the simple passage of time in consolidating this type of learning, with (wake rest group) or without (quiet rest group) input from the environment. We predicted that all groups would demonstrate a significant improvement in performance following the initial training session, but, as suggested by the findings on the finger-tapping learning following sleep (Korman et al., 2007; Nishida \& Walker, 2007), only the participants subjected to short and long naps were expected to show delayed performance gains.

\section{Method}

\section{Participants}

A total of 35 healthy volunteers between 20 and 35 years of age (mean age: $26 \pm 4.4$ years; 18 women) took part in this study. All were right-handed, as assessed by the Edinburgh Handedness Inventory (Oldfield, 1971). They reported sleeping regularly between 7 and $9 \mathrm{~h}$ per night. Extreme evening- and morning-type individuals, as well as regular nappers and smokers, were excluded. None of them had neurological, psychiatric, or sleep disorders or any prior history of drug or alcohol abuse, and they were instructed to be drug, alcohol, and caffeine free for $24 \mathrm{~h}$ prior to and during the experiment. Musicians and professional typists were excluded to avoid participants with previous experience on finger-tapping sequence tasks. This study was approved by the Research Ethics Committee of the University of Pisa, and all participants signed an informed consent form. The procedure used in this experiment was explained, and instructions regarding the motor task and questionnaires were given, but no information was provided about the objectives of the study or the dependent variables of interest.

\section{Design and apparatus}

Motor tasks To compare the effects of different offline conditions on the MI consolidation of motor sequence learning and to identify the sleep stage correlates mediating the consolidation process of MI practice, between-subjects (long/short nap-wake/quiet) and within-subjects conditions (pretest/posttraining/retest) were implemented. A computerized version of the sequential finger-tapping task developed by Karni et al. (1995) was used to measure motor sequence learning. This task was chosen because rapid 
changes in behavioral performance are usually observed with practice and because it provides robust sleepdependent consolidation effects (Fischer, Hallschmid, Elsner, \& Born, 2002; Korman et al., 2007). Moreover, Rodriguez, Llanos, Gonzalez, and Sabate (2008) have shown that both physical and imagined finger motor sequences are performed at a similar pace. Participants were first asked to memorize a sequence of eight moves employing fingers 2 to 5 (i.e., $2-4-3-5-3-4-2-5$ ), using their nondominant hand, until they were able to perform it explicitly from memory. The order of finger movements was pseudorandomly selected such that each finger was used twice in a sequence. They were requested to tap the sequence as quickly and accurately as possible on a computer keyboard during periods lasting $30 \mathrm{~s}$, with as few errors as possible. The participants were requested to push the space bar of the computer after completing the sequence, in order to record its duration. All keypresses were recorded and averaged over the entire sequence, using a homemade MATLAB-written routine. For each participant, this software compared the sequence of keypresses produced by the participant with the correct sequence template to be performed, hence allowing the detection of any discordance between the real and expected taps within the given sequence. Each 30 -s period was then followed by a rest period of $20 \mathrm{~s}$. The number of correct sequences and the average speed used to perform each correct sequence were the dependent variables of interest.

Sleep characteristics and MI abilities All participants filled out the Pittsburg Sleep Quality Index (Buysse, Reynolds, Monk, \& Timothy, 1989) to assess sleep quality and quantity. This test was administered to exclude participants who experience obvious disturbances during their sleep/wakefulness cycles and to ascertain participants' predisposition to benefit from the natural effects of sleep. Subjective measures of alertness and fatigue were also collected using the Stanford Sleepiness Scale (SSS; Hoddes, Dement, \& Zarcone, 1972) during the day preceding the experiment, as well as on the experimental day. In regard to MI ability, the revised version of the Movement Imagery Questionnaire (MIQ-R; Hall \& Martin, 1997) was administered to measure the individual ability to form kinesthetic and visual mental images. The test is an eight-item self-report questionnaire, in which participants have to rate the vividness of their mental representation using two 7-point scales. The first series of items measures the ability to form visual images $(1=$ very hard to see and $7=$ very easy to see), while the second rates the ability to perceive the sensations usually elicited by the movement during kinesthetic imagery $(1=$ very hard to feel and $7=$ very easy to feel). The participants also filled out the recent revised version of the Vividness of Movement
Imagery Questionnaire (VMIQ-2; Roberts, Callow, Hardy, Markland, \& Bringer, 2008) to determine (on a 5-point scale) the clarity with which they mentally imagine movements and, especially, the difference between their capacity to use internal and external visual imagery.

Pretraining session The experiment was scheduled to begin at 11:00. As was mentioned above, all participants were first asked to learn an explicitly known sequence of eight finger movements using their nondominant hand. To familiarize them with the sequence, they were given a few trials until they were able to physically perform five successive correct finger sequences. Practicing too many trials to learn the motor sequence (more than 10) was an exclusion criterion to ensure that the number of trials necessary to achieve five correct sequences did not differ among groups. Practically, none of the tested participants was excluded in this study. Following this introductory session, the pretraining session included four practice blocks lasting $30 \mathrm{~s}$ each, during which the participants physically performed the sequence as quickly and accurately as possible without visual feedback.

MI training The participants were randomly assigned to one of the four groups that differed in the type of nap or no-nap condition (Fig. 1): long-nap ( $n=11$, long), short nap ( $n=10$, short), quiet rest ( $n=6$, rest), and wake rest ( $n=8$, wake). All of the participants were subjected to MI training immediately after the pretest and were retested on the same day at 16:00. The training sessions did not include any actual repetition such that the sleep benefit could be attributed to MI practice. Furthermore, the sleep benefit could not be due to the initial learning achieved through the $2 \mathrm{~min}$ of actual practice of the finger sequence during the pretraining session. To ensure that performance during the learning and consolidation phases would not depend on individual imagery abilities, we verified that MIQ-R and VMIQ-2 test scores did not significantly differ among the four groups. During MI practice, all participants were required to imagine the finger sequence during 12 blocks of $30 \mathrm{~s}$ each, which were separated by 20 -s rest periods, for a total duration of $9 \mathrm{~min}$. The participants were required to imagine the motor sequence learning task using a combination of internal visual imagery and kinesthetic imagery-that is, imagining movement from within one's body and perceiving the sensations of how it feels to perform the action. They rehearsed the finger sequence in a quiet room without any environmental constraints - that is, without distracting stimuli-to facilitate focused attention on the formation of the mental images. On the basis of previous research (Debarnot et al., 2009a, b; Debarnot et al., 2010), a validated imagery script was read to the participants to ensure that they followed similar instructions throughout the MI session. To prevent actual 
Fig. 1 Schematic diagram of the experimental protocol. Experimental groups and times of the first session (including pretraining, imagery training, and immediate posttraining performance test) and nap conditions at $2 \mathrm{~h}$ following the initial learning, as well as retest session, are shown. Wake, active wake group; quiet, quiet rest group; short, short nap group; long, long nap group

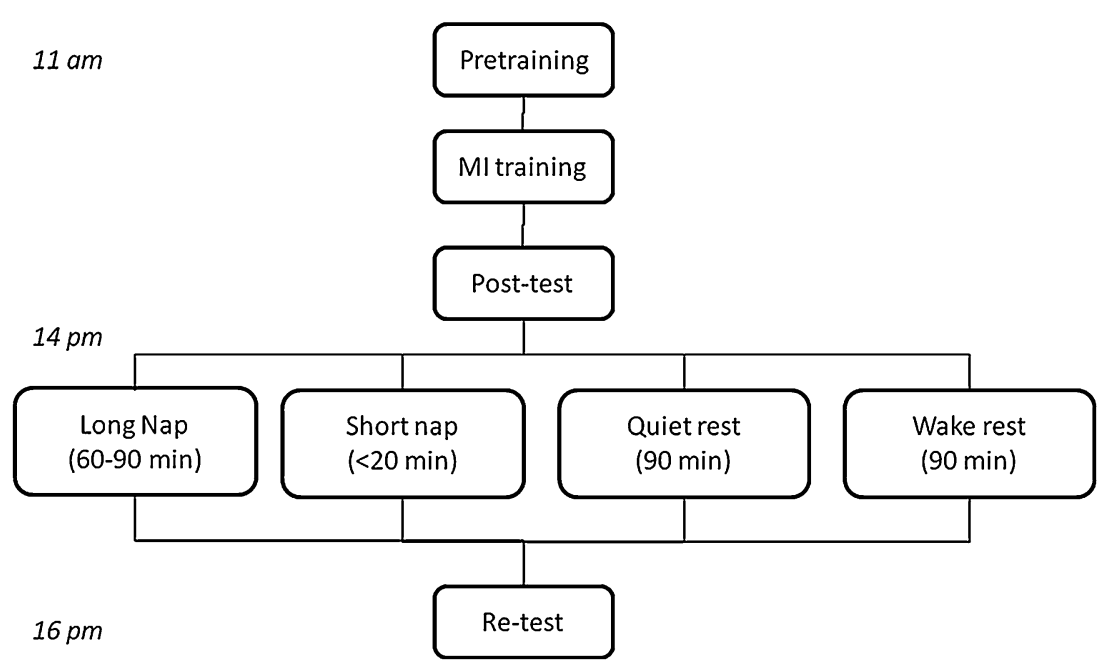

finger movements during MI, the participants were required to leave their left hand motionless on their right forearm and were asked to keep their eyes open to read the instructions on the computer screen. To make sure that all participants would follow these guidelines and to be able to record the duration of each sequence, they were asked to push the space bar with their right hand after imagining each motor sequence.

Posttraining and retest sessions Two test sessions were carried out to investigate the impact of MI practice, as well as the nap- (short and long), wake-, or quiet-related effects on motor memory consolidation. The first posttraining test was scheduled right after the MI training session and was similar to the pretraining test (i.e., four 30 -s periods during which the participants were asked to physically perform the finger sequence as quickly and accurately as possible). Individual debriefings were further scheduled in all groups to investigate adherence to the MI instructions and to determine whether the participants encountered difficulty in forming mental images. Accordingly, participants were asked to auto-evaluate the quality of their imagery, using a 6-point Likert-type scale (from $1=$ unclear and unvivid mental representation to $6=$ very accurate and vivid mental representation).

Finally, a second identical posttraining session (retest) was administered following the nap or no-nap (rest) conditions at 16:00.

Nap sleep determination and polysomnographic controls At 14:00, the participants were randomly assigned to one of the four groups, where they were subjected to different nap or no-nap conditions. Except for the wake rest group, polysomnographic activity was continuously recorded in all participants. Standard electroencephalographic (32-ch for EEG, 8-ch for auxiliary signals), electro-oculographic and electromyographic recordings were used to determine the total sleep time, as well as the duration of each sleep stage. Signals were acquired with a sampling rate of $1 \mathrm{kHz}$. Electrode impedance was below $5 \mathrm{k} \Omega$. Scalp EEG signals were referenced to the $\mathrm{FCz}$ potential. Participants from the nap conditions were allowed to sleep until they had completed either 10 or 60-90 min of polysomnographically identified sleep (Rechtschaffen \& Kales, 1968) and were then woken up by the experimenter. The signals were displayed on a computer monitor and rated visually, epoch by epoch, as NREM stages 1-4, REM sleep, rest, or movement time. In each nap condition, the nap period began at the first of three consecutive epochs below the $50 \%$ alpha baseline. In the short nap condition, participants were awakened after $10 \mathrm{~min}$ of sleep stage 2 since the first occurrence of a sleep spindle or K-complex. In the long nap condition, participants were awakened following a nap that ranged from 60 to $90 \mathrm{~min}$ and included both SWS and REM phases. Sleep stages were subsequently rescored offline. In the quiet rest condition, the participants were instructed to relax without falling asleep during a time lapse of $90 \mathrm{~min}$ in order to reduce interference effects that naturally occur during normal waking. They were blindfolded to prevent visual stimulations. Each participant's wake state was monitored, and the participants were alerted at the first indication of impending sleep. Finally, participants from the wake rest group were subjected to a period of neutral activities with the experimenter (card games) during equivalent time, without performing physical practice or MI at all. Such neutral activity could not interfere with the MI task, since it did not require the same cognitive abilities as those needed to imagine the motor sequence.

\section{Data analysis}

The dependent variables were the mean number of correct sequences and mean movement times - that is, time to 
complete each correct eight-digit sequence. Imagined times were also recorded to check whether participants complied with imagery guidelines. For the statistical analyses, we first checked that all data fitted a normal distribution and that there were no group differences during the pretraining test performance. Since no significant gender difference was found in both the number of correct sequences and their average times, we did not consider gender as an independent variable. Then two-way (group $\times$ session) repeated measures analyses of variance (ANOVAs) with Bonferroni post hoc corrections were performed to compare the data in all groups. In a first step, we tested for differences between short and long nap groups, as well as between quiet and wake rest groups. Then the main effect of being subjected to a nap was tested. Group scores on questionnaires were also compared by using ANOVAs. The results are presented as means ( \pm standard errors $[S E \mathrm{~s}]$ ), and threshold for significance was set at $p<.05$.

\section{Results}

\section{Questionnaires}

The average sleep score, as measured by the Pittsburg Sleep Quality Index, was 2.65 (1), thus attesting for the "good quality" of sleep in all participants. There was no significant difference in the SSS ratings between sessions, $F(1,31)=$ $2.16, p=.15, \eta^{2}=.07$, or among groups, $F(3,31)=2.12, p=$ $.12, \eta^{2}=.17$. Mean MIQ-R scores $(S E)$ were 43.67 (2.27) in the short nap group, 41.45 (4.38) in the long nap group, 43.71 (2.36) in the quiet rest group, and 45 (4.26) in the wake rest group. The ANOVA revealed that there was no significant group difference, $F(3,31)=1.06, p=.37, \eta^{2}=$ .12 , thus guaranteeing homogeneity in terms of individual ability to elicit motor mental images. The average VMIQ-2 scores were 93.78 (3.92) in the short nap group, 78.36 (8.52) in the long nap group, 91.29 (5.52) in the quiet rest group, and 96.25 (8.04) in wake rest group. The ANOVA revealed that there was no group difference, $F(3,31)=0.82, p=.49$, $\eta^{2}=.09$. When considering the internal visual, external visual, and kinesthetic imagery scales, post hoc analyses revealed better external visual imagery scores, as compared to kinesthetic imagery $(p<.05)$, as well as better internal visual imagery scores than kinesthetic imagery scores $(p<$ $.001)$ in all participants, whole sample mean scores $(S E)$ being 29.50 (1.50), 25.80 (1.21), and 35.27 (1.94) for the internal, visual, and kinesthetic imagery scales, respectively.

\section{Behavioral data}

There was no main effect of group when comparing the mean number of correct finger sequences during the pretraining session, $F(3,31)=0.45, p=.71$, hence demonstrating that the four groups did not differ in their ability to learn the finger sequence. Similarly, there was no difference when considering the individual average movement time to complete the sequence, $F(3,31)=1.10, p=$ .36 . We also verified that all groups of participants reached an asymptotic level of performance during the initial training session, hence showing that performance was stabilized before moving to the posttraining session.

The mean number of correct sequences during the pretraining session was 22.50 (1.42) in the short nap group, 24.82 (2.50) in the long nap group, 26.13 (2.63) in the quiet rest group, and 23.00 (3.40) in the wake rest group. They were 30.60 (1.31), 32.36 (2.78), 37.00 (4.31), and 29.50 (5.66) during the posttraining and 32.30 (1.27), 35.27 (3.03), 33.50 (4.41), and 28.50 (4.10) during the retest session, respectively.

A first statistical analysis was performed to compare the results obtained by the participants of the two nap groups, on the one hand, and those obtained by the two groups where participants were not subjected to sleep (quiet and wake rest groups), on the other. An ANOVA was first performed to see whether there was a different pattern of results in the groups where participants were subjected to either a short or a long nap. The ANOVA revealed a significant main effect of session, $F(2,38)=86.40, p<$ $.001, \eta^{2}=.82$, but no group effect, $F(1,19)=0.64, p=.43$, $\eta^{2}=.03$, and no group $\times$ session interaction, $F(2,38)=0.28$, $p=.76, \eta^{2}=.02$. Similarly, the ANOVA comparing the quiet and wake rest groups revealed a significant main effect of session, $F(2,24)=11.40, p<.001, \eta^{2}=.46$, but no group effect, $F(1,12)=0.91, p=.36, \eta^{2}=.02$, and no group $\times$ session interaction, $F(2,24)=0.62, p=.55, \eta^{2}=$ .04. On the basis of these data, participants subjected to either a short or a long nap were gathered into a single nap group, while participants who did not sleep (quiet and wake rest groups) were assigned into a single day group. Then a repeated measure ANOVA testing the effect of sleep yielded a significant main effect of session, $F(2,66)=55.61, p<$ $.001, \eta^{2}=.63$, as well as a significant group $\times$ session interaction, $F(2,66)=3.65, p<.05, \eta^{2}=.10$. Accordingly, further Bonferroni post hoc analyses revealed that both the nap and control rest groups improved their performance from the pretraining to the posttraining session $(p<.001)$, indicating that the outcome of MI training was comparable in all groups. Interestingly, data also revealed that the number of correct sequences further increased between posttraining and retest in both nap groups $(p<.001)$, while this effect was not observed in the control rest groups ( $p=$ .72; see Fig. 2).

A similar pattern of results was observed when movement times were used as a dependent measure. Mean movement times during the pretraining session were $3.80 \mathrm{~s}$ 
Fig. 2 Number of correct responses. A different pattern of results was found when the change in performance was compared between the posttraining test and the retest in participants who were subjected to either a short or a long nap, as compared to those in the rest control groups

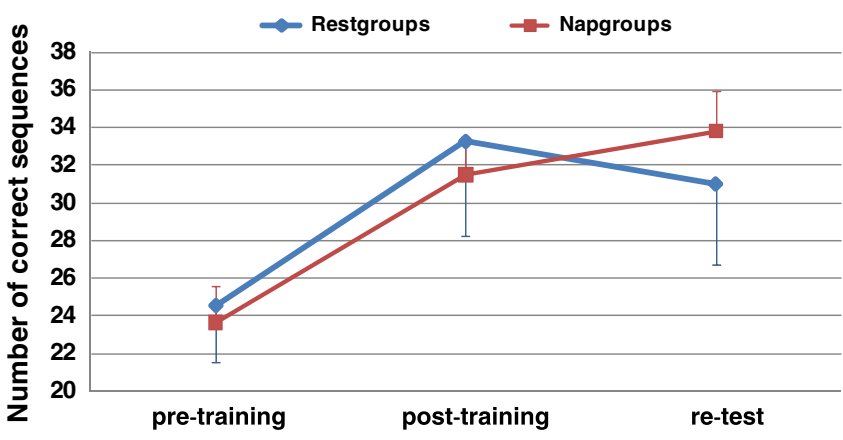

post hoc analyses showed that all groups improved their performance from pretraining to posttraining $(p<.001)$. Interestingly, participants subjected to a nap further improved their performance from the posttraining test to the retest $(p<.001)$, while such a performance gain was not seen in participants from the rest groups $(p=1$; see Fig. 3).

\section{Sleep data}

Polysomnography was performed to determine the total sleep time and to control the duration of each sleep stage. The mean $(S D)$ total sleep times were $19.4 \mathrm{~min}(2.5)$ in the short nap group and $66.4 \mathrm{~min}$ (9.3) in the long nap group (Table 1). More specifically, we checked that the duration of sleep stage 2 lasted $10 \mathrm{~min}$ in all participants from the short nap groups. We further controlled that the long nap of 60-90 min included both SWS and REM sleep stages. The two nap groups therefore differed with respect to the presence of SWS and REM sleep stages, while both included sleep stage 2 .

\section{Assessment of imagery use}

First, no significant group difference was found when comparing the participants' ratings in evaluating the vividness of their mental images during MI practice, $F(3$, $22)=0.39, p=.04, \eta^{2}=.07$. The mean score of the short nap group was $4.30(.21)$, while it was $4.54(.22)$ in the long nap group, 4.33 (.21) in the quiet rest group, and 4.33 (.33) in the wake rest group. Furthermore, during the debriefing following MI, all participants reported that they had used the imagery type outlined in the scripts. They adequately combined internal visual and kinesthetic imagery without switching to external visual imagery. None reported changing the imagery script to suit their individual needs, and all rehearsed the motor sequence as requested.

\section{Discussion}

The present study was designed to determine whether a nap following MI learning might contribute to the improvement 
Table 1 Characteristics of short and long naps. Mean times spent in each sleep stage during short and long naps are presented as min $\pm S E M$. S1, stage 1; S2, stage 2; SWS, slow wave sleep (stages 3 and 4); REM, rapid eye movement sleep

\begin{tabular}{llllll}
\hline & S1 $(\min )$ & S2 $(\min )$ & SWS $(\min )$ & REM (min) & Total Sleep Time \\
\hline Short nap & $9.4 \pm 2.5$ & 10 & - & - & $19.4 \pm 2.5$ \\
Long nap & $7.5 \pm 1.7$ & $18.1 \pm 4.2$ & $33.3 \pm 4.3$ & $7.4 \pm 3.1$ & $66.4 \pm 9.3$ \\
\hline
\end{tabular}

of motor memory consolidation in the same way as after physical practice and to identify which sleep stage might primarily mediate this consolidation process. Previous research provided strong evidence that performance enhancement following $\mathrm{MI}$ is sleep dependent and that the simple passage of time is not sufficient to provide additional benefits in participants who are not subjected to sleep (Debarnot et al., 2009b; Debarnot et al., 2010). Combined with the latter results, and most especially to the fact that the participants who did not perform MI practice did not improve their motor performance, the present findings showed that a daytime nap after MI practice improved motor performance and, therefore, facilitated motor memory consolidation, as compared to spending a similar time interval in the rest state. Interestingly, the results further revealed that both short and long naps resulted in similar delayed gains in performance, hence suggesting that the presence of SWS and REM sleep stages does not necessarily provide additional benefits for the sleep-dependent motor skill consolidation.

The data provided evidence that the number of correct motor sequences increased and that mean movement times were reduced after either a short or a long nap, while participants who were not subjected to a nap did not show delayed gains in performance. These findings support the role of sleep in the offline (re)processing of memories (Stickgold \& Walker, 2007), as shown previously by the great number of experimental studies reporting the existence of delayed gains in performance after a night of sleep, but not after a comparable time interval during daytime (Fischer, Nitschke, Melchert, Erdmann, \& Born, 2005; Karni et al., 1998; Korman et al., 2007; Korman, Raz, Flash, \& Karni, 2003; Kuriyama, Stickgold, \& Walker, 2004; Maquet, 2001). Lately, Lewis, Couch, and Walker (2010) reported that learning-related brain responses are modulated by brain state (wake or sleep) during retention. Especially, the responses in the cerebellum, striatum, and supplementary motor area increased across sleep and have been related to delayed gains in performance. By contrast, such neural substrates decreased across wake and did not result in any performance gains. Consistently, Debas et al. (2010) demonstrated that the striatal activity was significantly greater during the retest session in participants who were subjected to a sleep period. To date, it is still unknown whether the neural networks related to the improvement of the sequential motor learning following a whole night of sleep are identically activated during a midday nap. Further experimental investigation might certainly contribute to see whether substrate modulations during a night of sleep following MI demonstrate similar increasing activations, primarily located within the striatum, which would result in offline consolidation of a new sequence of finger movements. Such an assumption is in line with the great amount of research looking for clearer associations between sleep-dependent changes in the neuronal representation and behavior output measures of memory consolidation.

As well, the present data also confirm the sleeprelated effects on motor memory consolidation following MI practice (Debarnot et al., 2009a, b; Debarnot et al., 2010). There is now some evidence that daytime naps are favorable for the consolidation of procedural memory (Backhaus \& Junghanns, 2006; Mednick et al., 2003; Nishida \& Walker, 2007) and, therefore, promote the expression of delayed gains in sequential motor learning (Doyon et al., 2009), but the present study shows for the first time that daytime sleep might contribute to improve MI learning. On the basis of the effects mentioned above, we can reasonably assume that, for sequential motor learning, daytime sleep (even a short nap including sleep stage 2) after MI practice would result in similar motor memory consolidation to that following physical practice. This result therefore supports the functional equivalence between MI and motor performance, which has already been well established with regard to functional and temporal equivalences (e.g., Decety et al., 1994; Guillot \& Collet, 2005, 2008; Holmes \& Collins, 2001).

Interestingly, the same pattern of results was observed when comparing the effects of short versus long nap; that is, delayed performance gains were observed after daytime sleep but were not dependent on the duration of the nap. These data support the findings by Takahashi, Fukuda, and Arito (1998) who reported comparable alertness improvements $3 \mathrm{~h}$ after 15- and 45-min nap conditions. Brief afternoon naps were also found to be recuperative and as efficient as longer naps in terms of improved alertness and performance (Hayashi, Motoyoshi, \& Hori, 2005; Tietzel \& Lack, 2001, 2002). Hence, and although some authors have reported differences in performance gains following naps of different durations (Mednick et al., 2002), both short and 
long naps may impart substantial memory benefits, as compared with equivalent periods of wakefulness. Practically, our results extend such sleep-related effects to the consolidation of movements that have been learned through MI. Our two nap groups spent at least $10 \mathrm{~min}$ in sleep but differed with respect to the presence of sleep stages SWS and REM, since the short nap did not exceed 20 min and finished before the occurrence of deep SWS. The fact that delayed gains following MI practice were similar in these groups therefore extends the results by Nader and Smith (2003), Nishida and Walker (2007), and Korman et al. (2007), who emphasized the importance of NREM sleep including stage 2 for efficient motor consolidation.

As with all research, this study has some limitations that should be considered before drawing general conclusions. Previous data showed that physical and MI practice of a sequential finger-tapping task resulted in similar offline performance gains following a night of sleep (Debarnot et al., 2009), while participants not being subjected to one of these types of practice showed a stabilization of their motor performance after a night of sleep (Debarnot et al., 2009). On the basis of these findings, and since the primary aim of our study was to examine the specific nap-related effect following MI practice, we did not test a control sample of participants without any training session. Practically, one can therefore not totally exclude the possibility that the alteration of the performance for the rest groups could be partially related to fatigue. Subjective data, however, tended to suggest that fatigue was not an issue, since a nonsignificant difference was reported in the SSS scores at the retest session. Although we controlled sleep stages by polysomnographic recordings, the limited sample size in each nap group did not allow finding correlations between features of the sleep stages and performance. However, and since recent evidence has reinforced the particular role of spindle activity in motor memory consolidation by facilitating neuronal plasticity (during sleep stage 2; Barakat et al., 2010; Fogel \& Smith, 2006, 2011; Tamaki, Matsuoka, Nittono, \& Hori, 2008), we think that further investigations are needed to determine whether features of stage 2 (i.e., spindle) sleep are similarly modulated following MI practice. Finally, the statistical power was quite low in such a small-sample context, although small effect sizes in the control groups and high effect sizes in the nap groups tend to support our interpretations.

In a seminal article, Korman et al. (2007) explored the effects of daytime sleep on the two aspects of motor memory consolidation in finger opposition sequencelearning tasks - that is, susceptibility to interference and delayed gains. They notably concluded that a 90-min nap, immediately posttraining, substantially reduces the susceptibility to interference, with robust delayed gains expressed overnight, and that the provision of a posttraining nap should be considered for facilitating the learning of motor skills. On the other hand, the present study, as well as previous work (Debarnot et al., 2009; Debarnot et al., 2009), definitely supports the daytime or nighttime sleep contribution to motor memory consolidation after MI practice. These studies, however, focused mainly on the expression of delayed gains but did not take into consideration susceptibility to interference. In more recent research, Debarnot et al. (2010) demonstrated that MI practice resulted in less retroactive interference than did physical practice of the same task and outlined the relevance of the first night of sleep for the consolidation process following MI practice. Future studies should ideally combine all these approaches. In particular, determining the effects of both daytime and nighttime sleep after MI practice on susceptibility to interference and delayed gains, in a randomized experimental design including polysomnographic recordings, will therefore be the next experimental step and an exciting focus of research in coming years.

\section{Appendix}

The following guidelines were given in the imagery script: "attempt to imagine yourself doing the finger motor sequence with your eyes opened by visualizing the different movements as if you had a camera on your head - you see and feel only what you would see and feel if you were actually executing the sequence. Pay attention to each finger movement and make sure to respect the correct sequence by imagining it at the same pace than during the pre-training session. Try to keep the same speed throughout the entire sequence. Just feel yourself going through the different steps of the sequential motor action, keeping in mind the correct sequence".

\section{References}

Backhaus, J., \& Junghanns, K. (2006). Daytime naps improve procedural motor memory. Sleep Medicine, 7, 508-512.

Barakat, M., Doyon, J., Debas, K., Vandewalle, G., Morin, A., Poirier, G., et al. (2010). Fast and slow spindle involvement in the consolidation of a new motor sequence. Behavioural Brain Research, 217, 117-121.

Brooks, A., \& Lack, L. (2006). A brief afternoon nap following nocturnal sleep restriction: Which nap duration is most recuperative? Sleep, 29, 831-840.

Buysse, D. J., Reynolds, C. F., Monk, T. H., \& Timothy, H. (1989). The Pittsburgh sleep quality index: A new instrument for psychiatric practice and research. Journal of Psychiatric Research, 28, 193-213.

Debarnot, U., Creveaux, T., Collet, C., Gemignani, A., Massarelli, R., \& Guillot, A. (2009a). Sleep-related improvements in motor learning following mental practice. Brain and Cognition, 69, 398-405. 
Debarnot, U., Creveaux, T., Collet, C., \& Guillot, A. (2009b). Contribution of sleep to motor consolidation: A motor imagery study. Sleep, 32, 1559-1565.

Debarnot, U., Maley, L., De Rossi, D., \& Guillot, A. (2010). Motor interference does not impair the memory consolidation of imagined movements. Brain and Cognition, 74, 52-57.

Debas, K., Carrier, J., Orban, P., Barakat, M., Lungu, O., Vandewalle, G., \& Doyon, J. (2010). Brain plasticity related to the consolidation of motor sequence learning and motor adaptation.107, 17839-17844.

Decety, J. (1996). Do imagined and executed actions share the same neural substrate? Cognitive Brain Research, 3, 87-93.

Decety, J., Perani, D., Jeannerod, M., Bettinardi, V., Tadary, B., Woods, R., et al. (1994). Mapping motor representation with positron emission tomography. Nature, 371, 600-602.

Dhand, R., \& Sohal, H. (2006). Good sleep, bad sleep! The role of daytime naps in healthy adults. Current Opinion in Pulmonary Medicine, 2, 379-382.

Diekelmann, S., Wilhelm, I., \& Born, J. (2009). The whats and whens of sleep-dependent memory consolidation. Sleep Medicine Reviews, 13, 309-321.

Doyon, J., Korman, M., Morin, A., Dostie, V., Hadj Tahar, A., Benali, H., et al. (2009). Contribution of night and day sleep vs. simple passage of time to the consolidation of motor sequence and visuomotor adaptation learning. Experimental Brain Research, $195,15-26$.

Feltz, D. L., \& Landers, D. M. (1983). The effects of mental practice on motor skill learning and performance. A meta-analysis. International Journal of Sport Psychology, 5, 25-27.

Ficca, G., Axelsson, J., Mollicone, D. J., Muto, V., \& Vitiello, M. V. (2010). Naps, cognition and performance. Sleep Medicine Reviews, 14, 249-258.

Fischer, S., Hallschmid, M., Elsner, A. L., \& Born, J. (2002). Sleep forms memory for finger skills. Proceedings of the National Academy of Sciences, 99, 11987-11991.

Fischer, S., Nitschke, M., Melchert, U. H., Erdmann, C., \& Born, J. (2005). Motor memory consolidation in sleep shapes more effective neuronal representations. Journal of Neuroscience, 25, 11248-11255.

Fogel, S. M., \& Smith, C. T. (2006). Learning-dependent changes in sleep spindles and stage 2 sleep. Journal of Sleep Research, 15, 250-255.

Fogel, S. M., \& Smith, C. T. (2011). The function of the sleep spindle: A physiological index of intelligence and a mechanism for sleepdependent memory consolidation. Neuroscience and Biobehavioral Reviews, 35, 1154-1165.

Guillot, A., \& Collet, C. (2005). Duration of mentally simulated movement: A review. Journal of Motor Behavior, 37, 10-20.

Guillot, A., \& Collet, C. (2008). Construction of the motor imagery integrative model in sport: A review and theoretical investigation of motor imagery use. International Review of Sport and Exercise Psychology, 1, 32-44.

Hall, C., \& Martin, K. (1997). Measuring movement imagery abilities: A revision of the movement imagery questionnaire. Journal of Mental Imagery, 21, 143-154.

Hayashi, M., Watanabe, M., \& Hori, T. (1999). The effects of a $20 \mathrm{~min}$ nap in the mid-afternoon on mood, performance and EEG activity. Clinical Neurophysiology, 110, 272-279.

Hayashi, M., Motoyoshi, N., \& Hori, T. (2005). Recuperative power of a short daytime nap with or without stage 2 sleep. Sleep, 28 , 829-836.

Hoddes, E., Dement, W. C., \& Zarcone, V. (1972). The development and use of the Stanford sleepiness scale. Psychophysiology, 9, 150.

Holmes, P. S., \& Collins, D. J. (2001). The PETTLEP approach to motor imagery: A functional equivalence model for sport psychologists. Journal of Applied Sport Psychology, 13, 60-83.
Jeannerod, M. (1994). The representing brain: Neural correlates of motor intention and imagery. Behavioural Brain Science, 17, $187-245$.

Karni, A., Meyer, G., Jezzard, P., Adams, M. M., Turner, R., \& Ungerleider, L. G. (1995). Functional MRI evidence for adult motor cortex plasticity during motor skill learning. Nature, 377, $155-158$.

Karni, A., Meyer, G., Rey-Hipolito, C., Jezzard, P., Adams, M. M., Turner, R., et al. (1998). The acquisition of skilled motor performance: Fast and slow experience driven changes in primary motor cortex. Proceedings of the National Academy of Sciences, 95, 861-868.

Korman, M., Raz, N., Flash, T., \& Karni, A. (2003). Multiple shifts in the representation of a motor sequence during the acquisition of skilled performance. Proceedings of the National Academy of Sciences, 100, 12492-12497.

Korman, M., Doyon, J., Doljansky, J., Carrier, J., Dagan, Y., \& Karni, A. (2007). Daytime sleep condenses the time course of motor memory consolidation. Nature Neuroscience, 10, 1206-1213.

Kuriyama, K., Stickgold, R., \& Walker, M. P. (2004). Sleep-dependent learning and motor-skill complexity. Learning and Memory, 11, 705-713.

Lewis, P. A., Couch, T. J., \& Walker, M. P. (2010). Keeping time in your sleep: Overnight consolidation of temporal rhythm. Neuropsychologia, 49, 115-123.

Malouin, F., Belleville, S., Richards, C. L., Desrosiers, J., \& Doyon, J. (2004). Working memory and mental practice outcomes after stroke. Archives of Physical Medicine and Rehabilitation, 85, 177-183.

Maquet, P. (2001). Sleep, dreams and memory. Science, 294, 1048 1052.

Mednick, S., Nakayama, K., Cantero, J. L., Atienza, M., Levin, A. A., Pathak, N., et al. (2002). The restorative effect of naps on perceptual deterioration. Nature Neuroscience, 5, 677-681.

Mednick, S., Nakayama, K., \& Stickgold, R. (2003). Sleep-dependent learning: A nap is as good as a night. Nature Neuroscience, 6, 697-698.

Munzert, J., Lorey, B., \& Zentgraf, K. (2009). Cognitive motor processes: The role of motor imagery in the study of motor representations. Brain Research Reviews, 60, 306-326.

Nader, R., \& Smith, C. (2003). A role for stage 2 sleep in memory processing. In P. Maquet, C. Smith, \& R. Stickgold (Eds.), Sleep and brain plasticity (pp. 87-98). Oxford: Oxford University Press.

Nishida, M., \& Walker, M. P. (2007). Daytime naps, motor memory consolidation and regionally specific sleep spindles. PLoS ONE, 2, 341-371.

Oldfield, R. C. (1971). The assessment and analysis of handedness: The Edinburgh Inventory. Neuropsychology, 9, 97-113.

Rechtschaffen, A., \& Kales, A. (1968). A manual of standardized terminology, techniques and scoring system for sleep stages of human subjects. Bethesda, MD: U.S. Department of Health, Education and Welfare, Public Health Services.

Roberts, R., Callow, N., Hardy, L., Markland, D., \& Bringer, J. (2008). Movement imagery ability: Development and assessment of a revised version of the vividness of movement imagery questionnaire. Journal of Sport and Exercise Psychology, 30, 200-221.

Rodriguez, M., Llanos, C., Gonzalez, S., \& Sabate, M. (2008). How similar are motor imagery and movement? Behavioral Neuroscience, 4, 910-916.

Stickgold, R., \& Walker, M. P. (2007). Sleep-dependent memory consolidation and reconsolidation. Sleep Medicine, 8, 331343.

Takahashi, M., \& Arito, H. (2000). Maintenance of alertness and performance by a brief nap after lunch under prior sleep deficit. Sleep, 23, 813-819. 
Takahashi, M., Fukuda, H., \& Arito, H. (1998). Brief naps during post-lunch rest: Effects on alertness, performance, and autonomic balance. European Journal of Applied Physiology, 78, 93-98.

Tamaki, M., Matsuoka, T., Nittono, H., \& Hori, T. (2008). Fast sleep spindle $(13-15 \mathrm{~Hz})$ activity correlates with sleep-dependent improvement in visuomotor performance. Sleep, 31, 204-211.
Tietzel, A. J., \& Lack, L. C. (2001). The short-term benefits of brief and long naps following nocturnal sleep restriction. Sleep, 24, 293-300.

Tietzel, A. J., \& Lack, L. C. (2002). The recuperative value of brief and ultra-brief naps on alertness and cognitive performance. Journal of Sleep Research, 11, 213-218. 